\title{
Community awareness, engagement and linkage to care efforts by peer community-health workers to increase PrEP uptake among men who have sex with men in Baltimore, Maryland
}

\section{Jason E. Farley ( $\nabla$ jfarley1@jhu.edu )}

Johns Hopkins University School of Nursing https://orcid.org/0000-0002-6896-0134

Derek T Dangerfield II

Johns Hopkins University

Jessica LaRicci

Johns Hopkins University

Paul Sacamano

Johns Hopkins University

Omeid Heidari

Johns Hopkins University

Kelly Lowensen

Johns Hopkins University

Jacky Jennings

Johns Hopkins University

Karin Tobin

Johns Hopkins University

Research article

Keywords: PrEP, HIV prevention, Baltimore, men who have sex with men

Posted Date: March 2nd, 2020

DOI: https://doi.org/10.21203/rs.3.rs-15665/v1

License: (c) (i) This work is licensed under a Creative Commons Attribution 4.0 International License.

Read Full License 
Community awareness, engagement and linkage to care efforts by peer community-health workers to increase PrEP uptake among men who have sex with men in Baltimore, Maryland

Jason E. Farley ${ }^{1 \S}$, Derek T. Dangerfield $\mathrm{II}^{1}$, Jessica LaRicci ${ }^{1}$, Paul Sacamano ${ }^{1}$, Omeid Heidari ${ }^{1}$, Kelly Lowensen ${ }^{1}$, Jacky M. Jennings ${ }^{2}$, Karin Tobin ${ }^{3}$

1 The REACH Initiative, Johns Hopkins University School of Nursing, Baltimore, Maryland, 21205, USA

2 Center for Child and Community Health Research, Johns Hopkins University, Bloomberg School of Public Health, Baltimore, Maryland, 21205, USA

3 Department, Johns Hopkins University, Bloomberg School of Public Health, Baltimore, Maryland, 21205, USA

${ }^{\S}$ Corresponding author: Jason E. Farley, PhD, MPH, ANP-BC, FAAN, FAANP, AACRN

855 N. Wolfe Street

Rangos, Suite 601, Room 606, Mailbox \#30

Baltimore, Maryland, USA

410-258-4506

jfarley1@jhu.edu

E-mail addresses of authors:

JEF: jfarley1@jhu.edu

DTD: ddanger2@jhu.edu

JL: jlaricci@jhu.edu

PS : paull2@elrio.org

OH : oheidar1@jhu.edu

KL : klowens1@jhu.edu

JMJ : jennings@jhmi.edu

KT : ktobin2@jhu.edu

Keywords : PrEP; HIV prevention; Baltimore; men who have sex with men 


\section{Abstract}

2 Background: Despite clear evidence that pre-exposure prophylaxis (PrEP) prevents HIV, uptake

3 remains suboptimal across the United States, particularly in populations at greatest risk of HIV

4 infection, such as men who have sex with men (MSM) in Baltimore. This paper will evaluate

5 awareness, engagement and linkage to care for PrEP activities through multiple outreach

6 strategies.

7 Methods: This is an observational evaluation of the impact of LGBTQ+ outreach on the PrEP

8 cascade between March 1, 2016 to March 31, 2019. Descriptive statistics are used to characterize

9 the data and the linkage cascade by outreach type.

Results: Across all activities, our team had contact with 2,370 participants and scheduled a PrEP

11 appointment for $84(3.5 \%)$ with 58 (2.4\%) who completed the intake visits. Among 54 venue-

12 based activities, which focused on PrEP awareness messaging, 2,125 participants were reached

13 with $11.4 \%(243 / 2125)$ interested in further discussion with a peer community-health worker

14 (CHW). After 3 separate attempts to contact the individual, peer contact was successful in $66.2 \%$

$15(161 / 243) ; 11.2 \%(18 / 161)$ of those were interested in linkage to PrEP; $83.3 \%(15 / 18)$ had a

16 PrEP appointment scheduled; and 53.3\% (8/15) completed the PrEP visit. Online and telephone-

17 based outreach resulted in 24 contacts through prepmaryland.org and 43 calls on the PrEP warm

18 line, all of these indivduals reporting interest in further discussion with the peer $\mathrm{CHW}$. Among

19 prepmaryland.org users and warm line calls 54.2\% (13/24) and 67.4\% (29/43), respectively,

20 were successfully contacted, while 100\% (13/13) and 75.9\% (22/29) of those reported interest in

21 a PrEP referral. Among the referred, 61.5\% (8/13) and 86.4\% (19/22) had a PrEP appointment

22 scheduled; and 50\% (4/8) and 78.9\% (15/19) completed the visit. The mobile app, PrEPme,

23 yielded 178 unique downloads; $94.4 \%$ (168/178) had interest in further discussion with a peer 
24 CHW; follow-up contact was successful 64.3\% (108/168); 41.6\% (45/108) were interested in

25 PrEP referral; 95.5\% (43/45) had a PrEP appointment successfully scheduled; and 72.1\% (31/43)

26 completed the intake visit.

27 Conclusions: Outreach efforts reached a large number of participants attending LGBTQ+ centric

28 and health-based events, yet resulted in a relatively low total yield of engagement with peer

29 CHWs and even lower documented PrEP initiations.

30

31

32

33

34

35

36

37

38

39

40

41

42

43

44 


\section{Introduction}

46 In the United States, men who have sex with men (MSM) are at substantial risk for HIV infection

47 (1,2). In Baltimore, Maryland, most new HIV infections occur among African American and

48 Latino MSM less than 35 years of age (3). Despite clear evidence that pre-exposure prophylaxis

49 (PrEP) prevents HIV, PrEP uptake remains suboptimal across the U.S., particularly in priority 50 populations at greatest risk of HIV infection, such as MSM in Baltimore (4,5). Although PrEP 51 awareness has increased among MSM generally, awareness is associated with higher levels of 52 education, older age, Caucasian race, and social network connections and norms (6-8), while many 53 at-risk MSM in Baltimore are younger and from socio-economically disadvantaged communities. 54

55 Facilitators of PrEP initiation among MSM include access to sexual health services, sex-positive 56 counseling, peer networks, the ability to obtain PrEP outside of a primary care provider's office, 57 and perceptions of higher personal HIV risk $(9,10)$. Barriers include misinformation about HIV risk, PrEP stigma, concerns of potential side effects, and costs (11-14). These barriers intersect for MSM of color who have heightened medical mistrust $(6,15)$, greater internalized homonegativity

$60(13,16)$, greater experiences of racism in healthcare settings $(4,16)$ and within the gay community

61 (17), as well as lower social-network engagement in PrEP (18-21). Peer-based interventions in 62 which a self-identified gay or bisexual male facilitates awareness, patient navigation, care 63 coordination and culturally congruent support are increasing in the U.S.. Peer navigation by 64 community-health workers (CHW) helps overcome some of the barriers identified above, 65 improves PrEP initiation and adherence, and reduces cost in research settings $(22,23)$. However, 66 the optimal community-based approaches, including the setting and type of peer-based activities 67 that translate into effective linkage and engagement remains limited. 
While some have described that community health activities are helpful to engage MSM (24), few

69 have quantified peer-based community outreach efforts and assessed the relative yield of 70 participation and impact on PrEP initiation in real-world settings. Since 2016, our peer-led, 71 community-health worker team has participated in awareness, engagement, and linkage to care

72 activities to encourage PrEP initiation among MSM in Baltimore, Maryland. The objective of this 73 paper is to evaluate the impact of multiple outreach activities on the PrEP care cascade including 74 awareness, engagement and linkage to care among MSM. This work occurred alongside a 75 citywide campaign (The IMPACT Collaborative), the focus of which was to participate in events 76 and activities to increase awareness and willingness to engage in PrEP services.

77

\section{Methods}

79 This observational evaluation explores the impact of outreach activities on the PrEP care cascade 80 between March 1, 2016 to March 31, 2019 in Baltimore, Maryland as part of a public health 81 practice initiative. Outreach was conducted by trained peer $\mathrm{CHW}$ sy The REACH Initiative, a 82 center within The Johns Hopkins University School of Nursing. Activities involved educational 83 outreach, HIV testing, and a status neutral approach. Linkage to PrEP and/or HIV care services 84 were offered based on interest and/or need. Our team sought to perform outreach at community85 based events focused on the lesbian, gay, bisexual, transgder and queer (LGBTQ+) community to 86 increase PrEP awareness and HIV prevention messaging. We also engaged in three forms of 87 virtual, participant-initiated activities (i.e., a web-based contact portal on prepmaryland.org, a 88 PrEP telephone warm line and the PrEPme smartphone application). Throughout the process, peer 89 CHWs tracked their outreach efforts using a HIV prevention cascade. 


\section{Preparation for peer navigation by community-health workers (peer CHW):}

92 Peer CHWs were less than 25 years old, and belonged to the African American LGBTQ+ community in Baltimore City. Educational backgrounds ranged from high school diplomas to

94 bachelor preparation. Peers had no formal health training, but participated in a 12-month, 4session, competency-based PrEP CHW curriculum that integrated adult learning principles and case-based presentations during their first year of employment. Each training session was offered by members of the IMPACT collaborative group with expertise on the respective PrEP topic with coordinationi by The REACH Initiative of The Johns Hopkins University School of Nursing. Peer CHWs engaged in an initial 3-day intensive training to immediately prepare them for outreach and PrEP navigation activities followed by quarterly booster training sessions. Our referral network of PrEP providers included locations diverse in geographic setting, income requirements, which included free service provision in some sites, age appropriate care for young adults, and LGBTQ service providers. Transportant assistance to PrEP visits was offered as needed. Peer CHWs were also trained to assist with co-pay assistance programs to further reduce any associated costs.

\section{Evaluation methods and definitions:}

107 Descriptive statistics including counts, frequencies and proportions are used to describe the PrEP 108 linkage to care cascade by outreach activity type. To accomplish linkage at venue-based activities, peer CHWs sought voluntary sign-up either through a paper-based sign-in sheet or a tablet-based

110 enrollment feature of the mobile smartphone application, PrEPme®. This process was designed 111 to facilitate further peer CHW contact for the purposes of discussing PrEP and/or HIV in a one112 on-one, private session. The sign-up sheet may or may not have included a real name, did not 113 identify HIV status and only required a primary form of contact (i.e., phone or email address). 
114 Individuals wanting follow-up contact, voluntarily provided this information. Within 48 hours a 115 peer CHW would contact the individual based upon their preferred contact method. Individuals 116 who contacted the team through the website, warmline or PrEPme app would provide the same 117 contact details and receive a one-on-one follow-up with the peer CHW when contact was 118 successful.

Definitions for the PrEP Care Cascade:

121 There were five steps to the Peer CHW outreach process for tracking PrEP Care Cascade outcomes. 122 These steps included:

123 1. Approached. Approached refers to participants having a brief (1-3 minute) conversation with an outreach team member, either initiated by the team member or the participant. As this level of discussion did not require any personal information or health history, all individuals who had this brief-awareness conversation were counted including individuals who knew they were living with HIV and openly shared this information.

2. Interested in follow-up contact. Individuals were given an opportunity to speak privately with a peer $\mathrm{CHW}$ about PrEP and HIV prevention during or after all awareness activities. Options for follow-up contact included the following: phone call, face-to-face meeting, email, text, or app-based chat feature within PrEPme. After an outreach event, a peer navigator would attempt contact via an individual's chosen approach. If a peer CHW communicated with the person in a private session, the linkage step was changed to "contacted." Three attempts were made to reach participants before the individual was deemed "not contacted." To avoid breaches of confidentiality, peer CHWs left generic, scripted voice mail messages for individuals who preferred to be contacted by phone, text, 
or email. Contact through the PrEPme chat is protected and encrypted using standards set forth in HIPPA.

3. Interested in Linkage. After the private session, which included education, counseling, risk assessment, and a question/answer session, individuals were asked if they would like a PrEP referral. If they were interested in being linked directly by the peer $\mathrm{CHW}$, the individual's continuum status was changed to "interested in linkage". Individuals not interested in referral or those interested in self-navigation would be identified as "not interested in referral."

4. Appointment scheduled. Individuals were asked to work with the peer $\mathrm{CHW}$ to provide details about their insurance status and clinic preferences for appointment scheduling. Information was collected through telephone discussions or uploaded images of insurance cards using PrEPme. Persons without insurance were also provided linkage to PrEP care and/or research studies offering free clinical services, as well as, referrals to clinic-based health insurance navigators. Once a PrEP intake appointment was scheduled, the individual's linkage status was changed to "appointment scheduled."

5. Completed intake. To meet this step in the continuum, peers had to verify attendance at a scheduled PrEP intake appointment through follow-up with the individual. If follow-up contact was successful and the patient self-reported or the clinic confirmed completing the PrEP intake, this met the definition of "completed intake." 


\section{Results}

Across all forms of outreach activities, our team had contact with 2,370 participants in Baltimore with PrEP appointments scheduled for 84 (3.5\%) and 58 (2.4\%) who completed the PrEP intake visit.

The team participated in 54 community-based outreach activities, which reached 2,125 participants resulting in $11.4 \%(243 / 2,125)$ with reported interest in further discussion with a peer CHW. After three separate attempts to contact the individual through their preferred method, peer contact was successful in $66.2 \%(161 / 243) ; 11.2 \%(18 / 161)$ were interested in peer CHW assistance with linkage to PrEP; $83.3 \%$ (15/18) actively worked with the peer CHW and had a PrEP appointment successfully scheduled; and 53.3\% (8/15) completed the intake visit for PrEP. The overall success for community-based outreach activities was 8/2,125 (0.38\%).

Online and telephone-based outreach resulted in 24 contacts through prepmaryland.org and 43 telephone contacts on the PrEP warm line. All contacts in both groups (24/24 and 43/43) reported interest in being contacted by the peer CHW. Among prepmaryland.org users and warm line calls $54.2 \%(13 / 24)$ and $67.4 \%$ (29/43) were successfully contacted after three attempts, while $100 \%$ (13/13) and 75.9\% (22/29) of those reported interest in a PrEP referral. Among those referred, $61.5 \%(8 / 13)$ and $86.4 \%(19 / 22)$ actively worked with the peer CHW and had a PrEP appointment successfully scheduled; and 50\% (4/8) and 78.9\% (15/19) completed the intake visit for PrEP. The overall success for prepmaryland.org was 4/24 (16.7\%) and the PrEP warmline was 15/43 (34.9\%). The mobile app, PrEPme, yielded 178 unique downloads; 94.4\% (168/178) identified interest in further discussion with a peer CHW; follow-up contact was successful 64.3\% (108/168); 41.7\% 
183 (45/108) were interested in PrEP referral; 95.5\% (43/45) actively worked with the peer CHW and 184 had a PrEP appointment successfully scheduled; and 72.1\% (31/43) completed the intake visit for 185 PrEP. The overall success for PrEPme was 31/178 (17.4\%).

186 Figure 1. Is the peer community health worker (CHW) PrEP Care Cascade, Baltimore, MD

187 between March 2016 and March 2019.

188 Voluntary collection of demographic data was requested for individuals who attended the PrEP 189 intake visit with $26 / 58$ providing their demographic details. Race/ethnicity was reported as: 15/26 190 (57.7\%) African American/Black; 10/26 (38.5\%) Caucasian; 2/26 (7.7\%) Hispanic; and 1/26 191 (3.8\%) Asian. The majority, 22/26 (84.6\%) were male and reported sex with other men 19/22 $192(86.4 \%)$.

194 Discussion

195 This paper details three years of community outreach activities to increase PrEP awareness, 196 engagement, linkage to care and PrEP initiation among MSM in Baltimore City. Overall, outreach 197 efforts reached a large number of participants attending LGBTQ-centric and health-based 198 entertainment events, yet resulted in a relatively low yield of interest in one-on-one engagement 199 with peer CHWs and even lower documented PrEP initiations. While community-based outreach 200 activities had the lowest yield in PrEP intake visits within this evaluation $(<1 \%)$, those activities 201 also resulted in the largest community reach. Online and telephone-based outreach methods 202 produced higher yields interest in one-on-one discussions with a peer CHW, but substantially 203 fewer total follow-up contacts were successful compared to other methods. Peer CHWs were 204 successful at making contact with more than $50 \%$ of the individuals who expressed interest, 205 ranging from 54\% from online to $67 \%$ warm line. This suggests that having protocols to encourage 
multiple contact attempts is important for engagement and movement towards PrEP initiation.

207 When a peer CHW was able to have a warm line conversation, this method had the greatest overall success with $34 \%$ of individuals completing their PrEP intake visit. The PrEPme app chat and prepmaryland.org instant messaging features both resulted in fewer completed PrEP intake visits compared to one-on-one warm line conversations with $16.4 \%$ and $17.4 \%$ noted respectively, but were greater than the traditional venue-based outreach approaches we offered.

213 We did not monitor how many of the individuals who completed a peer CHW session chose to not 214 use the CHW for direct linkage. Differences in awareness about PrEP and levels of readiness to 215 make a PrEP intake appointment may explain the results. Individuals reached through a 216 community event may have lower awareness about PrEP compared to individuals who sought out

217 contact and who may be more likely to be comtemplating initation. Individuals contemplating

218 PrEP may use the CHW session as a method for gathering more information about the process and 219 not be ready to commit to direct linkage. The proportion scheduling a PrEP intake was fairly high 220 across methods among those who expressed a desire for the Peer CHW to link. Future programs 221 may consider identifying individual readiness for each step of the PrEP cascade and tailor activities 222 accordingly.

224 Engaging MSM in preventative healthcare measures and research is essential (25) yet engagement 225 in research has challenges that require additional efforts to ensure adequate participation, 226 particularly among priority populations (26-28). To overcome these challenges, our approach was 227 guided by recommendations from the literature. As such, we employeed a well-trained, peer CHW 228 team, which was predemoniately represented by self-identifed African American LGBTQ+ 
community members. The peer CHW team was supported by both nurses and nurse practitioners with years of experience in linkage to care and care navigation in this community. The team designed and initiated culturally tailored events in collaboration with the African American MSM community along with other LGBTQ+ community-based organizations. All online resources were designed to engage individuals who might be part of the same gender loving community, yet who do not consider themselves as gay or MSM and vetted with community stakeholders prior to launch. All outreach activities included details about PrEP for sexual and gender minority communities and the cis-gender, heterosexual community. Communities of color were represented across all forms of outreach material and across all forms of sexual expression and gender representations. We believe this inclusive approach contributed to our success in conducting a high number of community-based events in Baltimore. Further, as part of the comprehensive 12month training program, all peer CHWs were trained in the fundamental tennats of intersectionality (29) as well as trauma-informed care $(30,31)$. This training may have contributed to a relatively robust number of people who were willing to have follow-up one-on-one sessions with the peer CHWs. Unfortunately, once this conversation had completed, the immediate offer of PrEP linkage to care was not as successful. We believe this rests in the need for continued engagement, follow-up and ongoing communication with this community to move the individual readiness for change from contemplation to preparation and action (32).

Limitations of this evaluation of a public health practice program include the following. While most community outreach events focused on MSM of color, larger LGBTQ events were open to all members of the Baltimore community. This form of outreach is limited to persons who identify or feel comfortable being seen in such spaces. Similarly, all forms of our virtual outreach identify 
252 the word PrEP in the web address, phone number and smartphone application, which may limit

253 their use by certain community members. These issues may limit the generalizability of these data

254 for populations who may have HIV risk through same sex attraction, but who do not identify with

255 the LGBTQ community. We did not collect any demographic data at any outreach event and

256 therefore cannot make assertions about our reach into any specific community. However, this

257 approach facilitated opportunities to engage and educate all participants at an event. It was not 258 possible to determine the proportion of participants who could have self-navigated to PrEP services

259 after outreach without peer CHW assistance. Further, prepmaryland.org and the PrEPme app offer 260 details on how to self-navigate to the nearest PrEP provider by zipcode and we do anticipate that 261 self-directed navigation did occur after peer CHW interactions.

263 Conclusions

264 Future efforts should explore preferences for outreach and community engagement among MSM.

265 Efforts should include sufficient time to attempt to engage individuals at multiple encounters and 266 recognize individual-level readiness for activities at each step of the PrEP cascade. Competing 267 priorities and intersecting social determinants of health should be explored from the perspective 268 of the community as well as the peer CHW to determine ways to prioritize service delivery and 269 needs. Alternative strategies to recruiting and engaging priority communities should be considered 270 and further research is clearly needed to understand how to improve PrEP engagement. 


\section{$271 \quad$ List of abbreviations}

272 MSM. Men who have sex with men

273 PrEP. pre-exposure prophylaxis

274 CHW. community-health workers

275 LGTBQ+. Lesbian, gay, trangender, bisexual, queer +

\section{Declarations}

277 Ethical approval. Although this work involved public health practice designed to increase PrEP

278 linkage to care in collaboration with the Baltimore City Health Department, The Johns Hopkins

279 Institutional Review Board reviewed and approved the protocol (IRB\# 00212680).

280 Consent for publication. Not applicable.

281 Availability of data and materials. Not applicable.

282 Competing interests. JEF has received an independent investigator award and an unrestricted

283 educational grant from Gilead Science, Inc. Further, JEF, JL, KL have received Gilead support for

284 a study entitled, "A Comparative Effectiveness Demonstration Project for Linkage and Retention

285 in PrEP Care for Men who have Sex with Men” grant \# IN-US-276-4422. These funds were not

286 used as part of this work.

287 Funding. The authors would like to acknowledge the following funding sources: Gilead, Inc;

288 Baltimore City Health Department Early Intervention Services (EIS) Grant Supported by the Ryan

289 White Program; Health Department Demonstration Projects to Reduce HIV Infections and 
290 Improve Health Outcomes for Men Who Have Sex with Men of Color at Risk for and Living with

291 HIV Infection (Baltimore City Health Department Grant \#PS15-1506); and ViiV Healthcare.

292 Authors' contributions. JEF, KL - developed the grants supporting this work; JEF, KL, JL, PS 293 developed the training and outreach materials as well as trained all peer CHWs; JEF, DTD, OH, $294 \mathrm{JJ}, \mathrm{KT}$ - provided expertise in MSM outreach; all authors reviewed and contributed to the 295 development of this manuscript.

296 Acknowledgements. We would also like to thank the peer navigators who offered time and energy 297 on this project along with the people of Baltimore and The State of Maryland who engaged with 298 our team throughout this process.

299 Authors' information. JF is a Professor, Nurse Practitioner providing HIV care and PrEP services 300 and the Director of the REACH Initiative, a Johns Hopkins University School of Nursing Center 301 with a focus on multiple infectious diseases, including the HIV care and prevention cascades.

\section{References}

303 1. Centers for Disease Control and Prevention. Preexposure prophylaxis for the prevention of 304 HIV infection in the United States - 2017 update: a clinical practice guideline. Preexposure prophylaxis for the prevention of HIV infection in the United States - 2017 update: a clinical practice guideline. 2017.

307 2. Hess KL, Hu X, Lansky A, Mermin J, Hall HI. Lifetime risk of a diagnosis of HIV infection in the United States. In: Annals of Epidemiology. 2017.

309 3. HIV in Maryland, 2017 [Internet]. Baltimore; 2018. Available from: https://phpa.health.maryland.gov/OIDEOR/CHSE/SiteAssets/Pages/statistics/MarylandHIV-Fact-Sheet-2018.pdf

312 4. Fallon SA, Park JN, Ogbue CP, Flynn C, German D. Awareness and Acceptability of Pre- 
exposure HIV Prophylaxis Among Men Who have Sex with Men in Baltimore. AIDS Behav. 2017;

5. Daughtridge GW, Conyngham SC, Ramirez N, Koenig HC. I am men's health:

6. Eaton LA, Driffin DD, Smith H, Conway-Washington C, White D, Cherry C.

7. Hoots BE, Finlayson T, Nerlander L, Paz-Bailey G, Wortley P, Todd J, et al. Willingness

to Take, Use of, and Indications for Pre-exposure Prophylaxis Among Men Who Have Sex With Men - 20 US Cities, 2014. Clin Infect Dis. 2016;

8. Hosek SG, Lemos D, Hotton AL, Isabel Fernandez M, Telander K, Footer D, et al. An HIV intervention tailored for black young men who have sex with men in the House Ball Community. AIDS Care - Psychol Socio-Medical Asp AIDS/HIV. 2015;

9. Wade Taylor S, Mayer KH, Elsesser SM, Mimiaga MJ, O’Cleirigh C, Safren SA. Optimizing content for pre-exposure prophylaxis (PrEP) counseling for men who have sex with men: Perspectives of PrEP users and high-risk PrEP naïve men. AIDS Behav. 2014;

10. Underhill K, Morrow KM, Colleran CM, Holcomb R, Operario D, Calabrese SK, et al. Access to healthcare, HIV/STI testing, and preferred pre-exposure prophylaxis providers among men who have sex with men and men who engage in street-based sex work in the US. PLoS One. 2014;

11. Petroll AE, Walsh JL, Owczarzak JL, McAuliffe TL, Bogart LM, Kelly JA. PrEP Awareness, Familiarity, Comfort, and Prescribing Experience among US Primary Care Providers and HIV Specialists. AIDS Behav. 2017;

12. Bauermeister JA, Meanley S, Pingel E, Soler JH, Harper GW. PrEP awareness and perceived barriers among single young men who have sex with men. Curr HIV Res. 2013; 
13. Mayer KH, Wang L, Koblin B, Mannheimer S, Magnus M, Del Rio C, et al. Concomitant socioeconomic, behavioral, and biological factors associated with the disproportionate HIV infection burden among Black men who have sex with men in 6 U.S. cities. PLoS One. 2014;

14. Hannaford A, Lipshie-Williams M, Starrels JL, Arnsten JH, Rizzuto J, Cohen P, et al. The Use of Online Posts to Identify Barriers to and Facilitators of HIV Pre-exposure Prophylaxis (PrEP) Among Men Who Have Sex with Men: A Comparison to a Systematic Review of the Peer-Reviewed Literature. AIDS Behav. 2018;

15. Cahill S, Taylor SW, Elsesser SA, Mena L, Hickson DM, Mayer KH. Stigma, medical mistrust, and perceived racism may affect PrEP awareness and uptake in black compared to white gay and bisexual men in Jackson, Mississippi and Boston, Massachusetts. AIDS Care - Psychol Socio-Medical Asp AIDS/HIV. 2017;

16. Quinn K, Dickson-Gomez J, Zarwell M, Pearson B, Lewis M. “A Gay Man and a Doctor are Just like, a Recipe for Destruction": How Racism and Homonegativity in Healthcare Settings Influence PrEP Uptake Among Young Black MSM. AIDS Behav. 2018;

17. Haile R, Rowell-Cunsolo TL, Parker EA, Padilla MB, Hansen NB. An empirical test of racial/ethnic differences in perceived racism and affiliation with the gay community: Implications for HIV risk. J Soc Issues. 2014;

18. Krakower DS, Mimiaga MJ, Rosenberger JG, Novak DS, Mitty JA, White JM, et al. Limited awareness and low immediate uptake of pre-exposure prophylaxis among men who have sex with men using an internet social networking site. PLoS One. 2012;

19. Calabrese SK, Underhill K. How stigma surrounding the use of HIV preexposure prophylaxis undermines prevention and pleasure: A call to destigmatize "truvada whores." American Journal of Public Health. 2015.

20. Khanna AS, Schumm P, Schneider JA. Facebook network structure and awareness of preexposure prophylaxis among young men who have sex with men. Ann Epidemiol. 2017; 
21. Kuhns LM, Hotton AL, Schneider J, Garofalo R, Fujimoto K. Use of Pre-exposure Prophylaxis (PrEP) in Young Men Who Have Sex with Men is Associated with Race, Sexual Risk Behavior and Peer Network Size. AIDS Behav. 2017;

22. Wheeler D, Fields S, Nelson L, Wilton L, Watkins P, Hightow-Weidman L, et al. HPTN 073: Prep uptake and use by black men who have sex with men in 3 us cities. Conference on Retroviruses and Opportunistic Infections. 2016.

23. Wheeler DP, Fields SD, Beauchamp G, Chen YQ, Emel LM, Hightow-Weidman L, et al. Pre-exposure prophylaxis initiation and adherence among Black men who have sex with men (MSM) in three US cities: results from the HPTN 073 study. J Int AIDS Soc. 2019;

24. Young LE, Schumm P, Alon L, Bouris A, Ferreira M, Hill B, et al. PrEP Chicago: A randomized controlled peer change agent intervention to promote the adoption of preexposure prophylaxis for HIV prevention among young Black men who have sex with men. Clin Trials. 2018;

25. Mayer KH, Bekker LG, Stall R, Grulich AE, Colfax G, Lama JR. Comprehensive clinical care for men who have sex with men: An integrated approach. The Lancet. 2012.

26. White JJ, Dangerfield DT, Grieb SM. Methodological considerations for conducting focus groups in HIV prevention research among Black men who have sex with men. Public Health Nurs. 2019;

27. C. H-O, J.P. L, D.P. W, S.D. F. HPTN 073: Successful engagement of Black MSM into a culturally relevant clinical trial for pre-exposure prophylaxis. J Int AIDS Soc. 2016;

28. Wheeler DP, Lucas J, Wilton L, Nelson LE, Hucks-Ortiz C, Watson CC, et al. Building effective multilevel HIV prevention partnerships with Black men who have sex with men: experience from HPTN 073, a pre-exposure prophylaxis study in three US cities. J Int AIDS Soc. 2018;

29. Bowleg L. "Once You've Blended the Cake, You Can't Take the Parts Back to the Main Ingredients": Black Gay and Bisexual Men's Descriptions and Experiences of Intersectionality. Sex Roles. 2013; 
394 30. Eaton LA, Driffin DD, Kegler C, Smith H, Conway-Washington C, White D, et al. The 395 role of stigma and medical mistrust in the routine health care engagement of black men who have sex with men. Am J Public Health. 2015;

397 31. Fields EL, Bogart LM, Galvan FH, Wagner GJ, Klein DJ, Schuster MA. Association of discrimination-related trauma with sexual risk among HIV-positive African American men who have sex with men. Am J Public Health. 2013;

400 32. Parsons JT, Rendina HJ, Lassiter JM, Whitfield THF, Starks TJ, Grov C, et al. Uptake of 401 HIV pre-exposure prophylaxis (PrEP) in a national cohort of gay and bisexual men in the United States: The Motivational PrEP Cascade HHS Public Access. J Acquir Immune Defic Syndr. 2017;

404

405

Figures

406 Figure 1. Legend of table: Excludes total outreach of 2,370. 
Figures

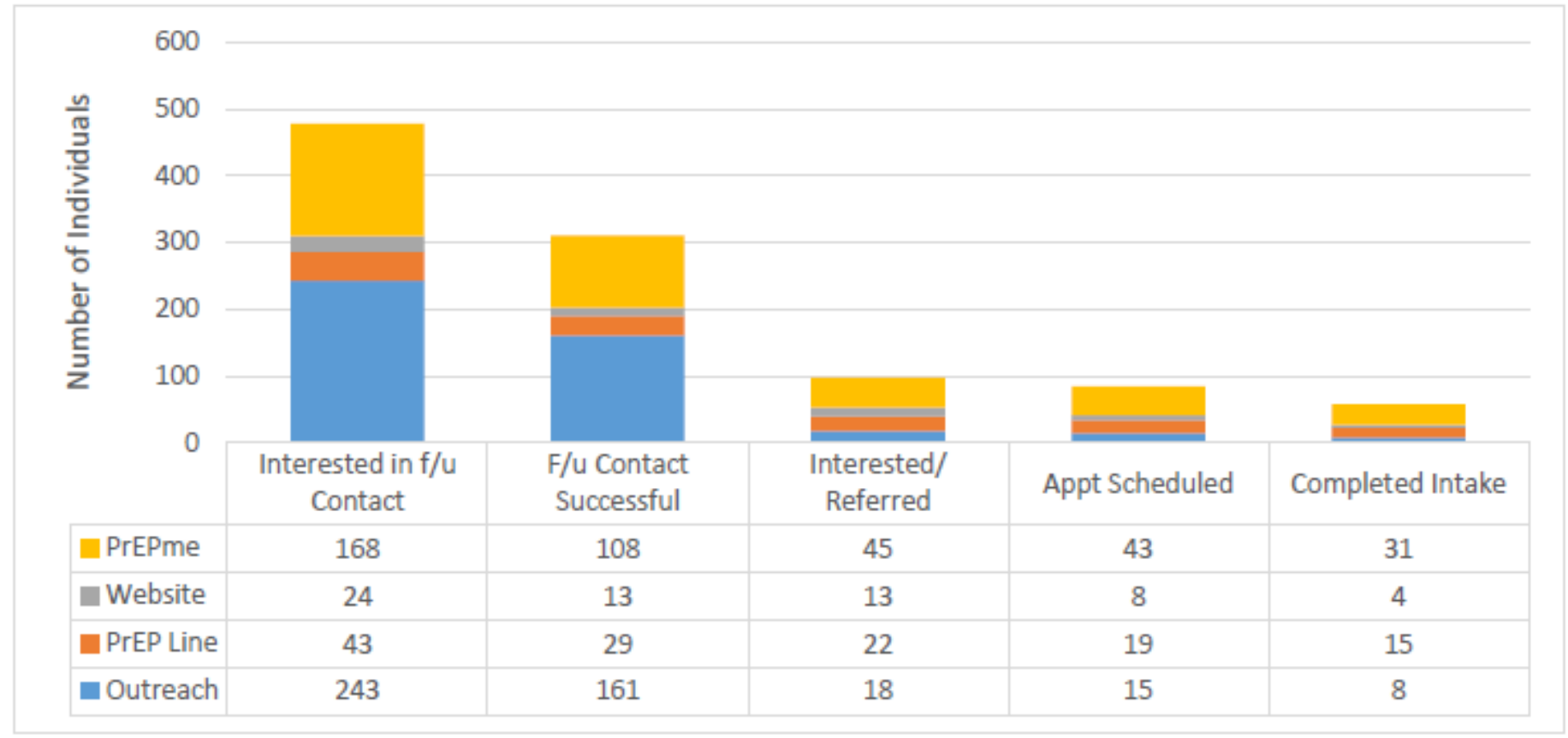

Legend of table: Excludes total outreach of 2,370.

\section{Figure 1}

Peer Community Health Worker (CHW) PrEP Care Cascade, Baltimore, MD between March 2016 and March 2019 